\title{
Pathogenic role of delta 2 tubulin in bortezomib-induced peripheral neuropathy
}

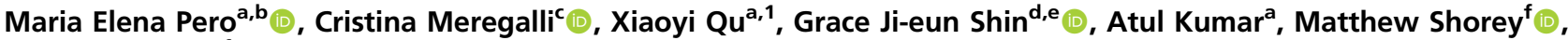

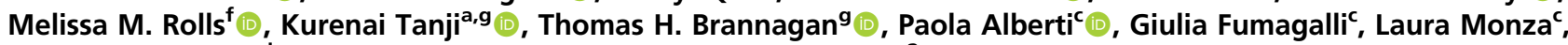

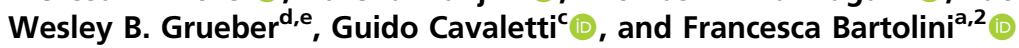

\begin{abstract}
${ }^{a}$ Department of Pathology and Cell Biology, Columbia University Medical Center, New York, NY 10032; ${ }^{b}$ Department of Veterinary Medicine and Animal Production, University of Naples Federico II, 80137, Naples, Italy; ' ${ }^{C}$ xperimental Neurology Unit, School of Medicine and Surgery and NeuroMI (Milan Center for Neuroscience), University of Milano-Bicocca, 20900, Monza, Italy; ${ }^{d}$ Department of Physiology and Cellular Biophysics, Zuckerman Mind Brain and

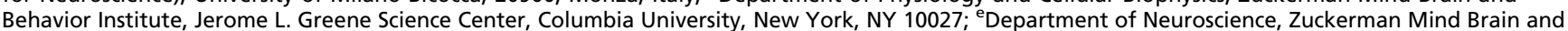
Behavior Institute, Jerome L. Greene Science Center, Columbia University, New York, NY 10027; ' Department of Biochemistry and Molecular Biology, Penn State University, University Park, PA 16802; and ${ }^{9}$ Department of Neurology, Columbia University Medical Center, New York, NY 10032
\end{abstract}

Edited by Yuh Nung Jan, Howard Hughes Medical Institute, University of California, San Francisco, CA, and approved December 3, 2020 (received for review June 21, 2020)

The pathogenesis of chemotherapy-induced peripheral neuropathy (CIPN) is poorly understood. Here, we report that the CIPN-causing drug bortezomib (Bort) promotes delta 2 tubulin (D2) accumulation while affecting microtubule stability and dynamics in sensory neurons in vitro and in vivo and that the accumulation of D2 is predominant in unmyelinated fibers and a hallmark of bortezomib-induced peripheral neuropathy (BIPN) in humans. Furthermore, while D2 overexpression was sufficient to cause axonopathy and inhibit mitochondria motility, reduction of D2 levels alleviated both axonal degeneration and the loss of mitochondria motility induced by Bort. Together, our data demonstrate that Bort, a compound structurally unrelated to tubulin poisons, affects the tubulin cytoskeleton in sensory neurons in vitro, in vivo, and in human tissue, indicating that the pathogenic mechanisms of seemingly unrelated CIPN drugs may converge on tubulin damage. The results reveal a previously unrecognized pathogenic role for D2 in BIPN that may occur through altered regulation of mitochondria motility.

delta 2 tubulin | mitochondria | DRG | bortezomib | axonopathy

C hemotherapy-induced peripheral neuropathy (CIPN) is a "dying back" neuropathy that features a distal-to-proximal peripheral nerve degeneration that frequently occurs in cancer patients undergoing chemotherapy (1). Modification of the chemotherapy treatment of a patient is sometimes required to limit the severity of CIPN, preventing patients from receiving effective cancer treatment (2). The pathogenesis of CIPN is largely unknown, and this incomplete knowledge is a main reason for the absence of effective neuroprotection strategies while maintaining chemotherapy drug anticancer activities (2-5). Several classes of anticancer drugs with different antineoplastic mechanisms can induce CIPN (6-8). However, sensory impairment is the predominant adverse effect associated with each class, suggesting the existence of a common mechanism of pathogenesis (2).

Tubulin and microtubules (MTs) are well-established targets for multiple anticancer drugs that induce CIPN (9). The contribution of the MT changes to the onset of CIPN is not well understood but is strongly implicated as the determining factor. MT hyperstabilization plays a direct role in paclitaxel neurotoxicity (10), and a single nucleotide polymorphism in TUBB2a, a gene encoding a tubulin isoform, is associated with an enhanced risk of CIPN (11). Moreover, MT dynamics and stability are influenced by nicotinamide adenine dinucleotide $(\mathrm{NAD}+)$ levels through sirtuin modulation (12), suggesting a tubulin-mediated mechanism also for the NAD+-consuming activity of Sterile Alpha and Tir Motifs-containing protein 1 (SARM1) in driving axonal degeneration in CIPN models (13).

Tubulin, its posttranslational modifications (PTMs), MT dynamics, and stability play critical roles in neurons via the regulation of long-distance transport, MT severing, $\mathrm{Ca}^{2+}$ homeostasis, and mitochondrial energetics (14-20). Each of these functions provides a potential mechanism that could result in the axonal degeneration in CIPN (21). Furthermore, tubulin and MTs functionally interact with Transient Receptor Potential Cation Channel Subfamily V (TRPV) members 1 and 4, two nonselective cation channels that are implicated in both sporadic and familial cases of peripheral neuropathy (22-24). Importantly, anomalies in MT dynamics and tubulin PTMs can drive axon regeneration failure and neurodegeneration (25-32), and $\alpha$-tubulin acetylation is a critical component of the mammalian mechanotransduction machinery and a pathological hallmark in the neuropathy induced by the drug vincristine and genetically inherited forms of peripheral neuropathy $(13,31,33-36)$.

In addition to taxanes and vinca alkaloids, tubulin changes have been reported downstream of the reversible $26 \mathrm{~S}$ proteasomal subunit inhibitor bortezomib (Bort), a widely employed drug with anti-tumor activity in hematological malignancies (37). Bortezomib-induced peripheral neuropathy (BIPN) is a painful

\section{Significance}

Chemotherapy-induced peripheral neuropathy (CIPN) is a debilitating "dying back" neuropathy featuring a distal-to-proximal peripheral nerve degeneration seen in cancer patients undergoing chemotherapy. The pathogenenic mechanisms of CIPN are largely unknown. We report that in sensory neurons, the CIPNinducing drug bortezomib caused axonopathy and disrupted mitochondria motility by increasing delta 2 tubulin (D2), the only irreversible tubulin posttranslational modification and a marker of hyper-stable microtubules. These data provide a new paradigm for the risk associated with enhanced tubulin longevity in peripheral neuropathy and suggest that targeting the enzymes regulating this tubulin modification may provide therapies that prevent the axonal injury observed in bortezomib-induced peripheral neuropathy.

Author contributions: M.E.P., G.C., and F.B. designed research; M.E.P., C.M., X.Q., G.J.-e.S., M.S., P.A., G.F., and L.M. performed research; M.E.P., C.M., X.Q., G.J.-e.S., A.K., M.S., M.M.R., K.T., T.H.B., W.B.G., G.C., and F.B. contributed new reagents/analytic tools; M.E.P., C.M., X.Q., G.J.-e.S., M.S., P.A., G.F., L.M., and F.B. analyzed data; and M.E.P. and F.B. wrote the paper.

The authors declare no competing interest.

This article is a PNAS Direct Submission.

This open access article is distributed under Creative Commons Attribution-NonCommercialNoDerivatives License 4.0 (CC BY-NC-ND).

1'Present address: Genentech, Inc., South San Francisco, CA 94080

${ }^{2}$ To whom correspondence may be addressed. Email: fb2131@columbia.edu.

This article contains supporting information online at https://www.pnas.org/lookup/suppl/ doi:10.1073/pnas.2012685118/-/DCSupplemental.

Published January 19, 2021. 
axonal sensory-predominant and length-dependent peripheral neuropathy that affects $\sim 40 \%$ of Bort-treated patients (3). Bort has been shown to increase MT polymer without disrupting MT ultrastructure and to affect MT-dependent axonal transport (37, 38). However, the in vivo and in vitro effects of Bort on tubulin PTMs and MT behavior as a potential BIPN pathogenic pathway have not been characterized. Furthermore, whether the perturbation of one or more tubulin PTMs may be sufficient and necessary to induce CIPN has not been determined.

Herein, we report that delta 2 tubulin (D2), an irreversible tubulin PTM residing on hyper-stable MTs, was increased in dorsal root ganglia (DRG) and sciatic nerves (SNs) from rats treated with Bort and in the sural nerve from a patient suffering from BIPN. D2 is a tubulin PTM that accumulates on very long-lived MTs and is promoted in vitro by MT stabilizers and drugs that cause CIPN, such as paclitaxel and vinca alkaloids $(39,40)$. The function of D2 remains unknown, although it is abundant in neurons as well as in long-lasting MT conformations in cilia. D2 is produced by the sequential action of a tubulin carboxypeptidase (VASH1/2) that cleaves the last residue of tyrosine from an $\alpha$-tubulin subunit residing on stable MTs followed by the irreversible cleavage of a residue of glutamic acid by carboxypeptidase 1 (CCP1), a member of a family of tubulin de-glutamylases that is also expressed in neurons (41-44). Tubulin tyrosine ligase (TTL) is a rate-limiting enzyme that re-tyrosinates tubulin and so controls the level of detyrosinated tubulin, a reversible short-lived D2 precursor (45). Interestingly, constitutive suppression of TTL in mice results in perinatal death linked to a disorganization of CNS neuronal networks, underscoring a critical role for de-tyrosination and D2 in neuronal development $(46,47)$ A pathogenic role for D2 in neurodegeneration, however, has never been demonstrated.

We found that Bort affects MT dynamics in vitro and in vivo and that D2 accumulation in sensory neurons may result from Bort-induced MT stabilization and increased tubulin heterodimer stability. Importantly, D2 was sufficient and necessary for both the axonopathy and disrupted mitochondria motility caused by Bort. These findings reveal a previously unrecognized role for the disruption of the tubulin tyrosination/de-tyrosination cycle in the onset of axonal injury that may occur through D2-dependent regulation of mitochondria transport.

\section{Results}

Bortezomib Induces D2 in BIPN. We measured tubulin PTM levels in the cell bodies of DRG and SNs isolated from rats that received acute and chronic administrations of Bort or untreated control (Fig. 1 and SI Appendix, Figs. S1-S3). In all cases, neuropathy was tested by mechanical and thermal nociception (SI Appendix, Fig. S1 $A$ and $B$ ). Notably, however, only the chronic regimen appeared to significantly affect behavior and nerve conduction, and the changes in conduction velocity and evoked response amplitude were more severe in the caudal than in the digital nerve (SI Appendix, Fig. S1B). We quantified the fate of selected tubulin PTMs (de-tyrosinated, polyglutamylated, acetylated, and D2 tubulin) in DRG and SNs isolated from chronically treated rats and found that they were not significantly affected, with the exception of de-tyrosinated and D2 tubulins which appeared to be slightly, yet significantly, reduced in DRG (Fig. $1 C$ and SI Appendix, Fig. S3). When we measured the fate of the same tubulin PTMs in tissues isolated from acutely treated rats prior to manifested neuronal injury or neuropathic behavior, however, we found that D2 was significantly increased in SNs and almost fivefold higher in the DRG cell bodies of Bort-treated rats than in controls (Fig. $1 A$ and $B$ and SI Appendix, Fig. S2). Furthermore, when classified by the two DRG neuron diameter markers NF200 and peripherin $(48,49)$, quantification of fluorescence intensity within the cell bodies revealed that D2 accumulation was higher within smallcaliber DRG neurons labeled by peripherin (Fig. $1 D$ and $E$ ), phenocopying the cell specificity of the damage to unmyelinated fibers observed in BIPN patients (50-52).

We tested whether D2 accumulation was promoted by Bort in human BIPN by examining an available sample from a sural nerve biopsy of a cancer patient affected by the disorder. D2 levels were increased in tissue sections and whole tissue lysates of the sural nerve biopsy from the patient with BIPN but not in three control patients suffering from unrelated neuropathies, suggesting conservation and specificity of the pathways that induce D2 by Bort (Fig. $1 F-H$ ).

We investigated whether D2 increase could be detected in vitro upon exposure of sensory neurons to pathogenic doses of Bort and analyzed how D2 levels correlated with axonal injury. To this end, adult mouse DRG neurons were grown in culture for $12 \mathrm{~d}$, to allow for full recovery from axotomy and axonal extension. The cultures were exposed to Bort for up to $72 \mathrm{~h}$ prior to quantifying relative D2 levels and axonal degeneration in individual axons (Fig. $2 A-C$ ). D2 was also detected by Western blot analysis of whole-cell (Fig. $2 D$ and $E$ ) and fractionated DRG lysates to evaluate total and partitioned D2 present in soluble and polymerized tubulin fractions (Fig. $2 F$ and $G$ ). We found that Bort promoted D2 accumulation and axonal injury in a time-dependent manner with a peak at $48 \mathrm{~h}$. Noticeably, a significant increase in D2 levels already occurred at $12 \mathrm{~h}$ at a dose that did not induce caspase-dependent cellular death up to 48 h (SI Appendix, Fig. S4).

Next, we assessed whether the increase in D2 was limited to the MT fraction or extended to the soluble pool. We found that while the bulk marker $\beta$ III-tubulin ( $\beta$ III) redistributed to the MT pellet in a time-dependent fashion, D2 accumulated with a similar kinetic in the soluble tubulin fraction. We hypothesized that this might be dependent on induction of MT stabilization and selective inhibition of soluble D2 turn over caused by loss of proteasome activity (Fig. $2 F$ and $G$ ). To examine this possibility directly, the consequences of Bort exposure on the stability of axonal MTs and levels of TTL and CCP1, two rate-limiting enzymes implicated in D2 generation, were measured in adult DRG neurons (12 days in vitro [DIV]) (Fig. $3 A-D$ and $S I A p$ pendix, Fig. S5 $A$ and $B$ ). Endogenous levels of CCP1 and TTL were only slightly affected even at $24 \mathrm{~h}$ of exposure, a time point at which significant D2 accumulation and axonal injury was observed (SI Appendix, Fig. S5 $A$ and $B$ and Fig. $2 A-E$ ). When we tested whether Bort could acutely perturb MT behavior, however, we observed that it promoted axonal MT stability, as measured by higher residual levels of $\beta$ III that were resistant to mild MT depolymerization induced by nocodazole in neurons treated with Bort for $6 \mathrm{~h}$ (Fig. $3 A$ and $B$ ). MT stabilization was further consistent with a time-dependent increase in acetylated and de-tyrosinated tubulins, two tubulin PTMs associated with enhanced MT longevity (Fig. $3 C$ and $D$ ).

We investigated whether MT stabilization resulted from acute perturbation of MT dynamics by measuring the behavior of EGFP-EB3 comets that only label the plus ends of polymerizing MTs (Fig. $3 E$ and $F$ and Movies S1 and S2). Bort led to timedependent inhibition of MT dynamics in cultured DRG neurons starting at $1 \mathrm{~h}$. We tested whether acute inhibition of MT dynamics also occurred in vivo by recording MT behavior in nociceptive neurons from adult zebrafish exposed to Bort by bath immersion and third-instar Drosophila larvae that had been fed on Bort-containing food (Fig. 4). An acute perturbation of MT behavior was observed in DRG neurons in adult zebrafish exposed to a dose of Bort that caused reversible degeneration of peripheral nerve endings at $96 \mathrm{~h}$ (Fig. $4 A-C$ ). Unlike the in vitro recordings, however, Bort did not seem to dampen MT dynamics in the nerve fibers of fish skin but rather only increased growth rates at 6 h (Fig. $4 B$ and $C$ and SI Appendix, Fig. S6 and Movies S3 and S4). To test MT behavior using a different model system, we set up an analogous experiment in Drosophila larvae fed on 
A

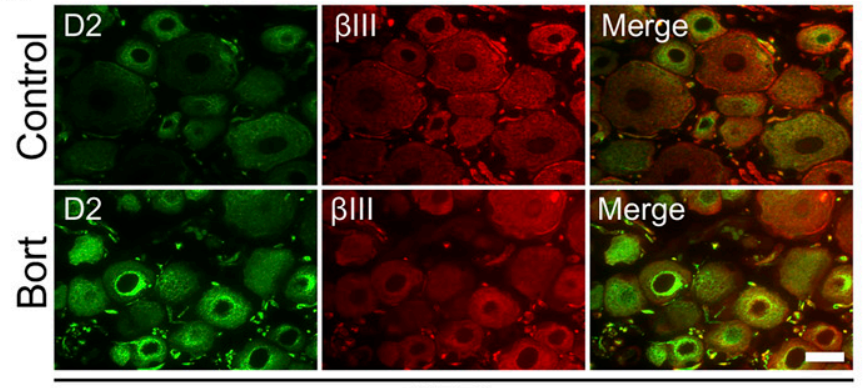

DRG

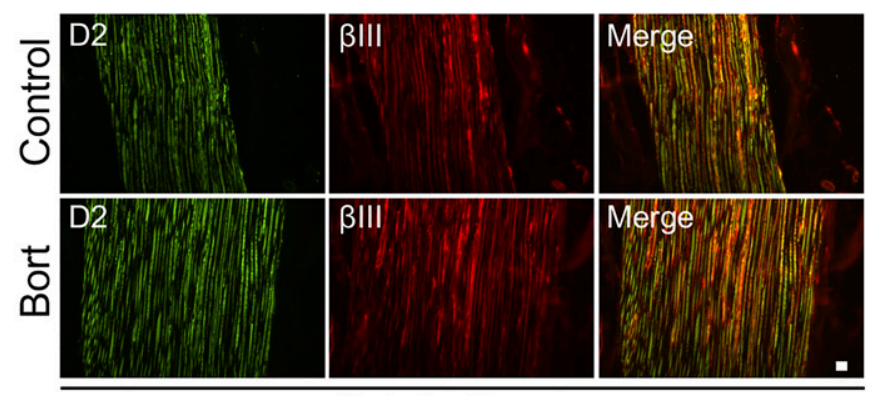

Sciatic Nerve

D
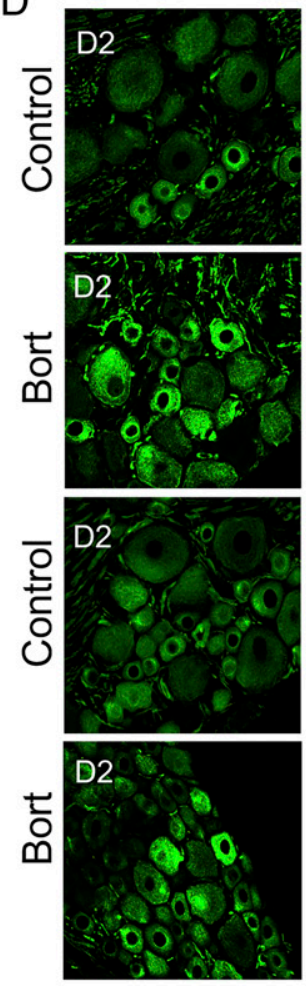
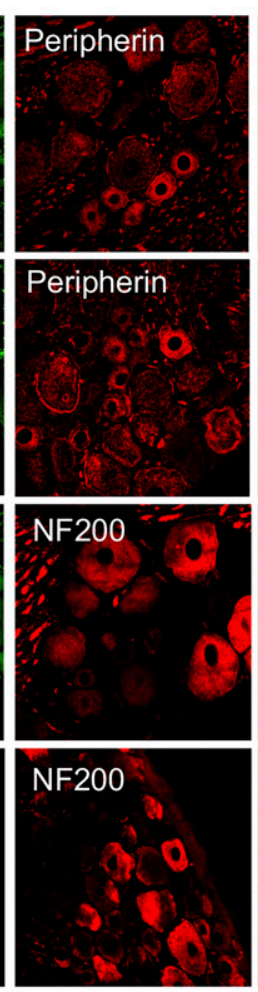

DRG
$\mathrm{F}$
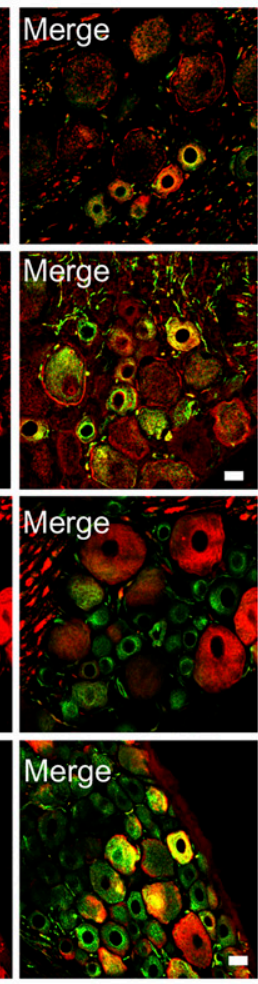

G
B

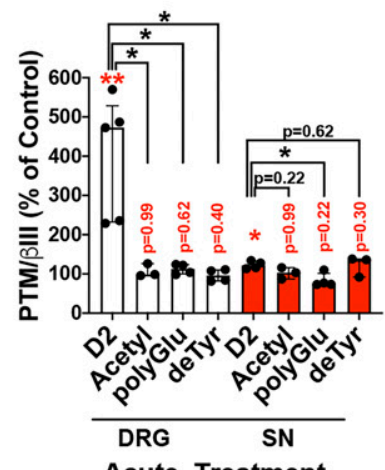

Acute Treatment
C

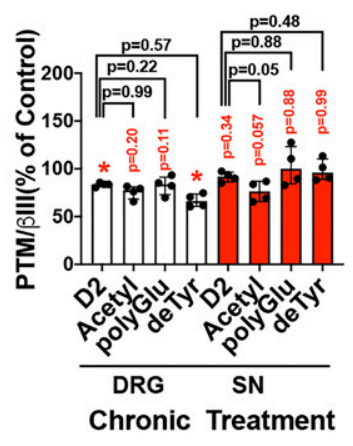

E
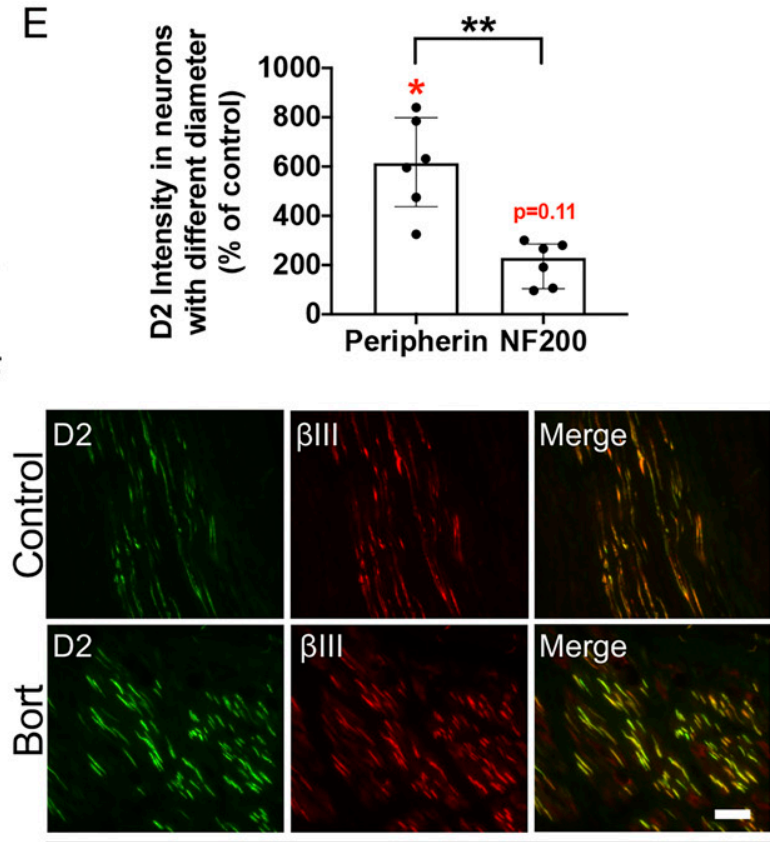

Sural Nerve

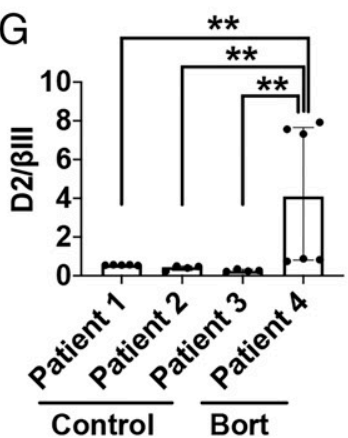

Fig. 1. Bort induces D2 levels in vivo. (A) Representative D2 and $\beta I I I I F$ staining of DRG cell bodies and the SN isolated from control rats or rats acutely treated with Bort (i.v. $0.2 \mathrm{mg} / \mathrm{kg} ; 24 \mathrm{~h}$ ). ( $B$ and C) Relative tubulin PTM levels measured by quantitative IF in randomly selected DRG cell bodies (35-60) and SN (five sections per condition) from rats treated with acute ( $n=3$ to 5 per group) (B) (i.v. $0.2 \mathrm{mg} / \mathrm{kg} ; 24 \mathrm{~h})$ or chronic $(n=4 \mathrm{per}$ group) (C) (0.2 mg/kg, $3 \times$ week for 8 wk) doses of Bort. $(D)$ Representative peripherin (smaller unmyelinated C-fibers), NF200 (medium and large myelinated A- $\beta$ fibers), and D2 IF staining of DRG cell bodies acutely treated with Bort. $(E)$ Relative D2 tubulin values measured in DRG cell bodies $(n=35$ to 60$)$ positive to either peripherin or NF200 from rats $(n=6)$ acutely treated with Bort (i.v. $0.2 \mathrm{mg} / \mathrm{kg} ; 24 \mathrm{~h})$. ( $F)$ Representative D2 and $\beta$ III IF staining of sural nerve biopsy from a patient with BIPN. (G) Ratio analysis of D2/ $\beta$ III levels measured by IF of fixed tissue (three to six sections per condition) from one sural nerve biopsy of a BIPN patient versus three sural nerve biopsies from control patients. $(H)$ Immunoblot analyses of D2 levels in whole cell lysates from one sural nerve biopsy from a patient treated with Bort displayed with a control patient. Tyr, tyrosinated tubulin. GADPH, loading control. All data in $B, C, E$, and $G$ are shown as medians plus interquartile range, and statistics were analyzed by Mann-Whitney $U$ test. ${ }^{*} P<0.05,{ }^{*} P<0.01$. Scale bars in $A, D$, and $F, 20 \mu$ m. Red writings are $P$ values when compared to control; black writings are $P$ values when compared between groups. 

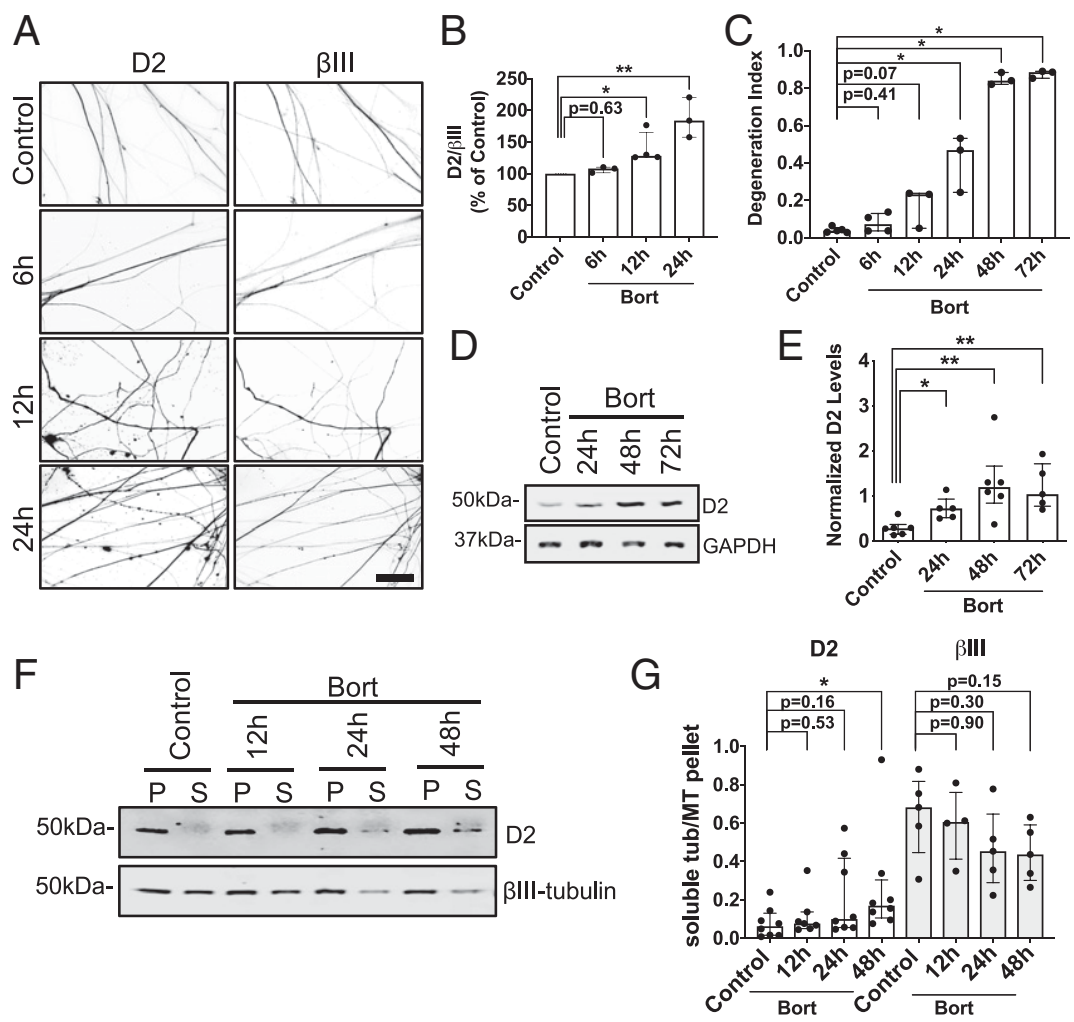

Fig. 2. Bort induces $D 2$ at the onset of axonal degeneration. (A) Representative IF images of $D 2$ and $\beta$ III staining in axons of DRG neurons (12 DIV) treated with $100 \mathrm{nM}$ of Bort for the indicated times. Scale bar, $50 \mu \mathrm{m}$. (B) Ratio analysis of D2/ $/ 1 \mathrm{ll}$ levels measured by IF in axons from fixed neurons treated as in $A$. Data are pooled from three to four experiments ( $n=10$ to 41 neurites per condition for each experiment). (C) Time-dependent increase of axonopathy in DRG neurons treated with $100 \mathrm{nM}$ of Bort for the indicated times. Data are from three to four experiments ( $n=4$ to 15 fields per condition for each experiment). $(D)$ Immunoblot analyses of D2 levels from whole cell lysates of adult DRG neurons (12 DIV) treated with increasing doses of Bort for the indicated times. (E) Quantification of normalized D2 levels as in D. Data are pooled from five to six experiments. (F) Immunoblot analyses of D2 and $\beta$ III levels present in the MT pellet (P) and soluble tubulin (S) fractions isolated from adult DRG neurons (12 DIV) and treated with $100 \mathrm{nM}$ of Bort for the indicated times. (G) Quantification of D2 and $\beta I I I$ levels as in $F$. Data are from four to eight experiments. All data in $B, C, E$, and $G$ are shown as medians plus interquartile range, and statistics were analyzed by Mann-Whitney $U$ test. ${ }^{\star} P<0.05,{ }^{*} P<0.01$.

Bort-containing food for up to $6 \mathrm{~h}$ at a concentration of the drug that caused significant degeneration of the dendritic arbors when Bort was administered chronically (Fig. $4 D$ and $E$ ). This system was adopted as an alternative to administration by bath immersion that may have an impact on the drug intracellular concentration. Similarly to cultured adult DRG neurons and unlike adult DRG neurons in zebrafish, we found that in Drosophila nociceptive neurons, Bort reduced catastrophe, rescue/nucleation frequencies and comet density at $3 \mathrm{~h}$ while affecting growth rates at $6 \mathrm{~h}$ (Fig. $4 F$ and $G$ and Movies S5 and S6). Because either inhibition of MT dynamics or increase in MT polymerization alone might lead to MT stabilization, our results indicate that administration of the drug to either cultured DRG neurons or nociceptive neurons residing in live tissue stabilizes MTs through acute perturbation of MT dynamics.

Together, these results demonstrate that in sensory neurons, Bort promotes D2 accumulation in vitro, in vivo, and in human tissue while acutely modifying MT behavior both in vitro and in vivo. They further indicate that in sensory neurons, D2 accumulation may result from Bort-dependent MT stabilization and increased tubulin heterodimer stability caused by proteasome inhibition.

D2 Accumulation Is Sufficient and Necessary to Cause Axonopathy. To test whether D2 accumulation induces axonopathy, lentiviral delivery of two independent TTL short hairpin RNA (shRNA) sequences was carried out in untreated adult DRG neurons starting at 5 DIV, to avoid any consequences of TTL loss on axon regeneration. Silencing of TTL was sufficient to increase both D2 levels and axonal fragmentation (Fig. $5 A-D$ ). To rule out tubulinindependent functions of TTL in causing axonopathy, D2 was expressed in untreated neurons and its effects on axonal integrity were evaluated. Wild-type (WT) $\alpha$-tubulin was also tested to control for undesired effects that expression of a tubulin subunit may have on cellular homeostasis.

Overexpression of recombinant D2 but not WT $\alpha$-tubulin dramatically promoted axonal degeneration in naive DRG neurons, suggesting a pathogenic role for accumulation of this irreversible tubulin PTM in the onset of BIPN (Fig. $5 E$ and $F$ ). To test this, we examined whether accumulation of D2 was necessary for Bortinduced axonopathy by administering Bort to adult DRG neurons that had been depleted of CCP1 expression. Lentiviral mediated depletion of CCP1 for either 7 or 10 d promoted substantial loss of D2 with no effects on polyglutamylated tubulin levels, a tubulin PTM also implicated in neurodegeneration and negatively regulated by the tubulin de-glutamylation activity of CCP1 $(25,28,29)$ (SI Appendix, Fig. S7 $A-C$ ). We elected to use the shortest silencing strategy $(7 \mathrm{~d})$ in order to minimize any toxic consequences of sustained CCP1 silencing on axonal integrity. Under these experimental conditions, we found no effect of reducing CCP1 levels on axonal integrity of untreated neurons (Fig. $6 A$ and $B$ ). We observed, however, that a fivefold increase in fragmentation caused by Bort in control cells was only twofold when CCP1 was knocked down, consistent with a pathogenic role for D2 accumulation in 

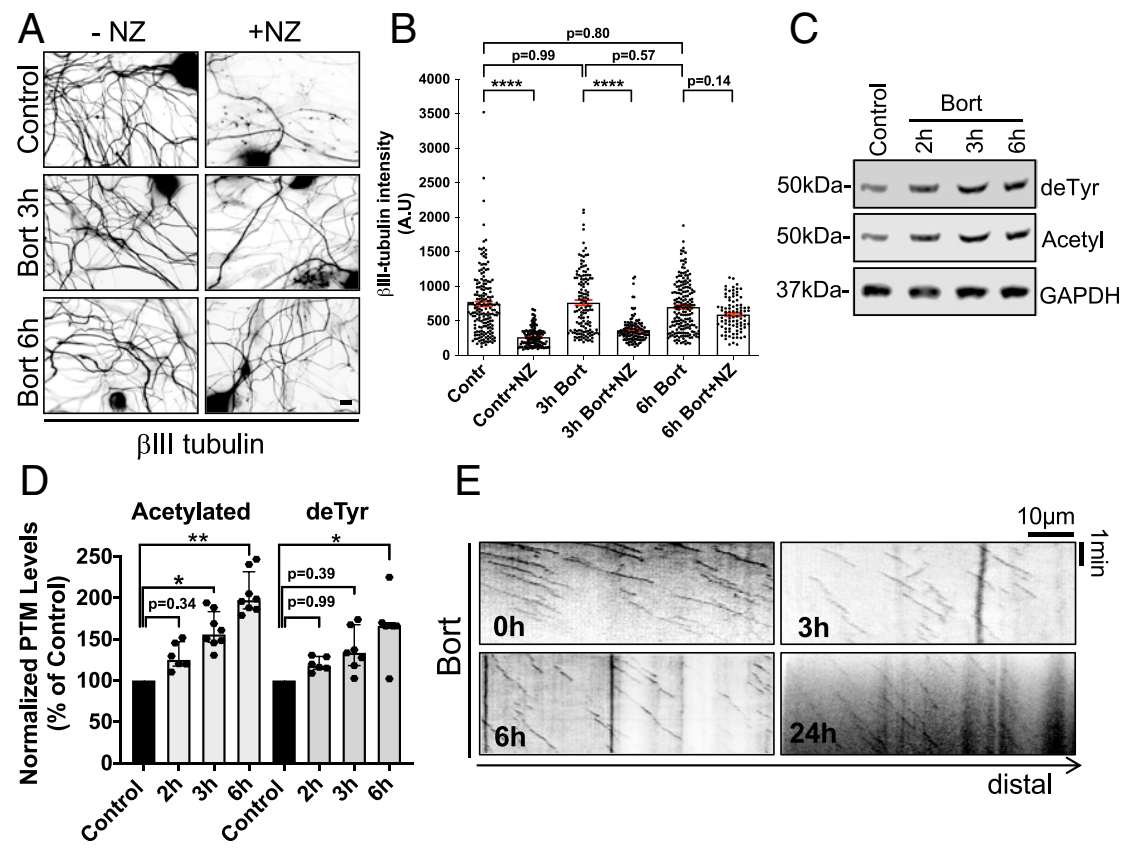

$\mathrm{E}$

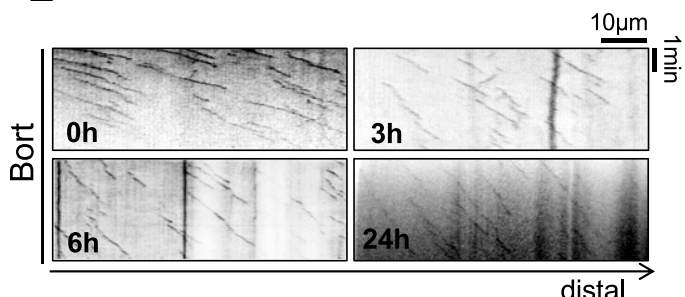

F
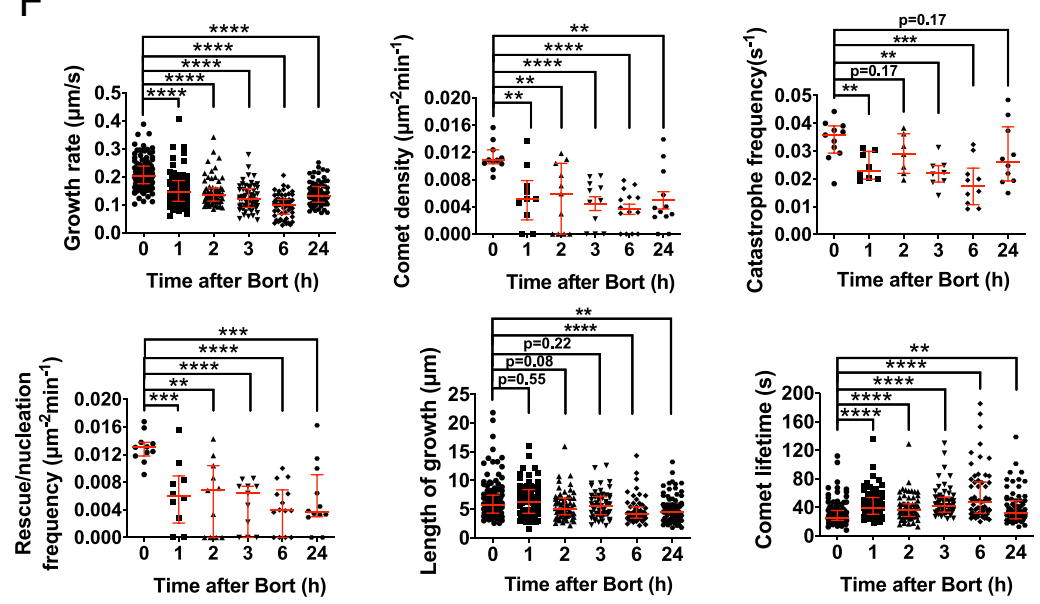

Fig. 3. Bort acutely induces MT stability and affects MT behavior in DRG neurons. (A) Representative IF images of $\beta$ III in adult DRG neurons (12 DIV), treated with $100 \mathrm{nM}$ of Bort for 3 and $6 \mathrm{~h}$, and incubated with $1 \mu \mathrm{g} / \mathrm{mL}$ nocodazole (NZ) for $45 \mathrm{~min}$ before MT extraction and fixation. Scale bar, $10 \mu \mathrm{m}$. (B) Quantification of residual MT mass in proximal axons (100- $\mu \mathrm{m}$ from cell bodies; 50 to 150 axons per time point) of DRG neurons treated as in A. All data are means \pm SEM and analyzed by ANOVA (multiple comparison). (C) Immunoblot analyses of de-tyrosinated (deTyr) and acetylated (Acetyl) tubulin levels in cultured 12 DIV treated with $100 \mathrm{nM}$ of Bort for 2 to $6 \mathrm{~h}$. (D) Quantification of normalized deTyr and Acetyl tubulin levels as in $C$. Data are from 6 to 8 experiments. (E) Representative kymographs at $0,3,6$, and $24 \mathrm{~h}$ of EB3-GFP comets in DRG axons treated with $100 \mathrm{nM}$ of Bort. Scale bar, $10 \mu \mathrm{m}$. $(F)$ An EGFPEB3 time course analysis of MT dynamics parameters in DRG neurons (12 DIV), treated as in $E$ for the indicated times. Data were from up to 118 comets and 7 to 11 neurites for each group. Data in $D$ and $F$ are shown as medians plus interquartile range, and statistics were analyzed by Mann-Whitney $U$ test. $* P<0.05$, $* * P<0.01, * * * P<0.001$, and $* * * * P<0.0001$

Bort-induced axonal injury (Fig. $6 A$ and $B$ and SI Appendix, Fig. S7 $G$ and $H$ ).

D2 Affects Mitochondria Motility. We explored whether the rescue observed in CCP1-depleted cells correlated with the ability of D2 accumulation to interfere with mitochondria motility, a hallmark of Bort-induced neurotoxicity in DRG neurons and a common feature of axonal degeneration (38). To this end, imaging of Mito-DsRed was conducted in cultured adult DRG neurons at 5 DIV, a time point at which mitochondria are motile and can be classified into subtypes according to mobility properties (Fig. 7).

We first investigated whether inhibition of motility by Bort was selective for mitochondria by imaging the dynamics of the lysosomal marker LAMP1-Cherry in cultured DRG neurons treated with Bort for $24 \mathrm{~h}$ (SI Appendix, Fig. S8 $A$ and $B$ and Movies S7 and S8). No perturbation in lysosomal motility was observed at Bort exposures that affect mitochondria motility, indicating a specific effect on mitochondria rather than on the overall MTdependent transport machinery.

We then tested whether D2 depletion was essential for inhibition of mitochondria motility caused by Bort. We found that acute depletion of CCP1 to an extent that affected D2 levels without significant effects on polyglutamylated tubulin levels (SI Appendix, Fig. S7 $D, E, F, I$, and $J$ ) ameliorated loss of mitochondria motility in Bort-treated DRG neurons, with limited selectivity for dynamic pausing mitochondria, characterized by constrained bidirectional motility, compared to anterograde (AR) or retrograde (RR) running mitochondria pools (Fig. $7 A$ and $B$ and Movies S9-S12). 

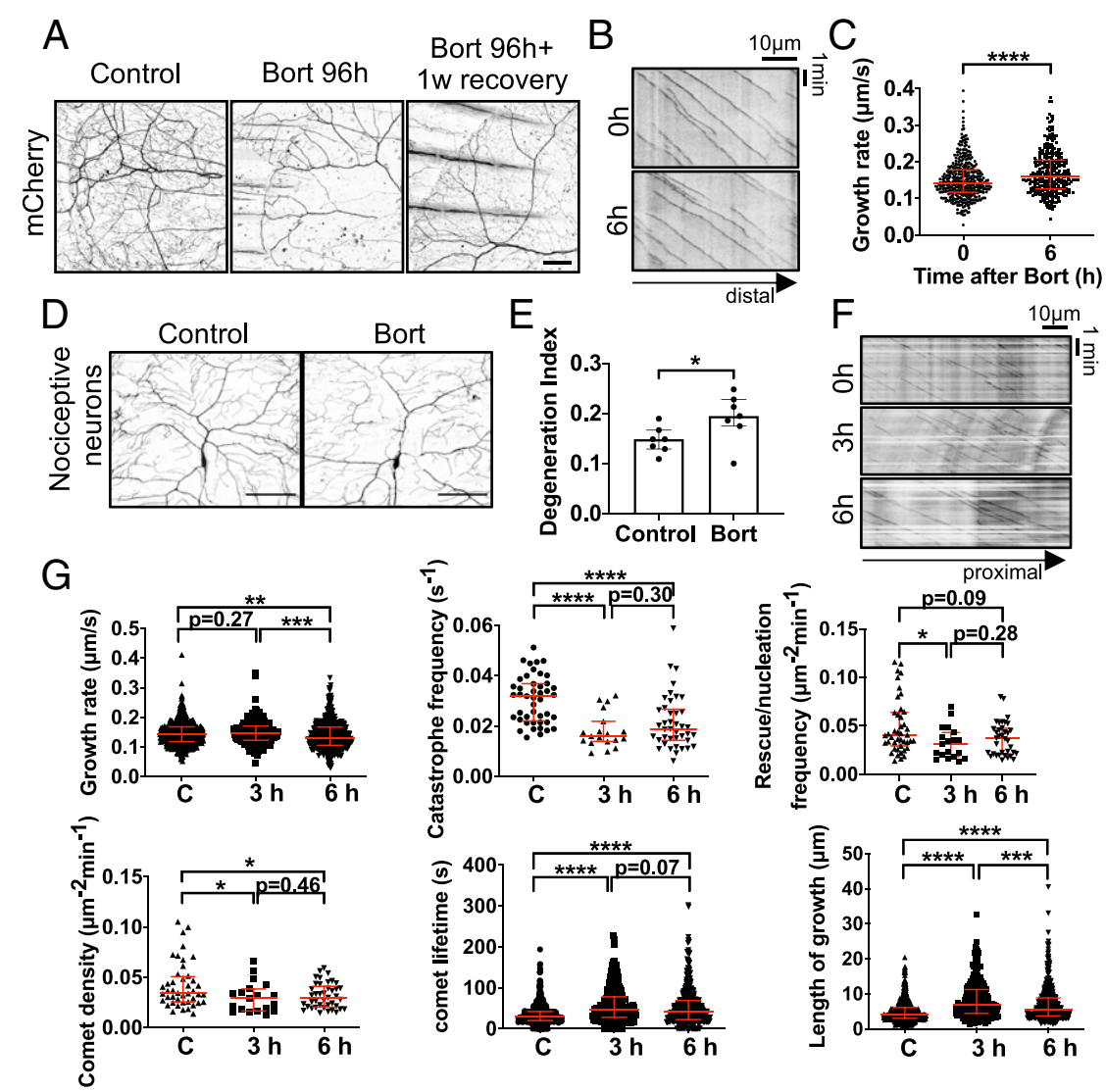

Fig. 4. Bort acutely affects MT behavior in DRG neurons in vivo. (A) Still images from live imaging of $m$ Cherry in the peripheral nerve fibers of zebrafish treated with $1.3 \mu \mathrm{M}$ Bort for the indicated times. Each image includes endings innervating the surface of a scale. Scale bar, $100 \mu \mathrm{m}$. (B) Representative kymographs at 0 and $6 \mathrm{~h}$ of EB3-GFP comets in the peripheral nerve fibers of zebrafish treated with Bort. (C) EB3-GFP time course analysis of MT dynamics parameters in the peripheral nerve fibers of zebrafish treated as in $B$ for the indicated times. Data were pooled from three individual fish containing four to five neurites with up to 80 to 220 comets. $(D)$ Micrographs from third-instar Drosophila larvae fed with vehicle control $(n=7$ animals, $n=28$ cells) or $20 \mu \mathrm{M}$ Bort ( $n=7$ animals, $n=45$ cells) starting 24 to $28 \mathrm{~h}$ after egg laying. ppk-CD4-tdGFP was used to visualize ddaC nociceptive neurons. (E) Quantification of dendrite degeneration treated as in $D$. Each point refers to an averaged value of degeneration index in each animal (4 to 6 cells per animal). Bars, $50 \mu \mathrm{m}$. ( $F$ ) Representative kymographs of EB1-GFP comets in control and nociceptive neurons of Drosophila larvae fed with Bort for 3 and 6 h. Scale bar, $10 \mu \mathrm{m}$. (G) EB1GFP time course analysis of MT dynamics parameters in nociceptive neurons of larvae treated for the indicated times. Data are from up to 400 comets and 19 to 45 neurites for each group. Control data are pooled from vehicle controls ( 3 to $6 \mathrm{~h}$ ). Data in $C, E$, and $G$ are medians plus interquartile range, and statistics were analyzed by Mann-Whitney $U$ test. ${ }^{*} P<0.05, * * P<0.01, * * * P<0.001$, and $* * * * P<0.0001$.

To investigate whether excessive D2 inhibited mitochondria motility per se, the effect of D2 or WT $\alpha$-tubulin overexpression was analyzed on mitochondria motility in untreated DRG neurons. While WT $\alpha$-tubulin overexpression did not significantly interfere with any class of motile mitochondria, overexpression of D2 alone inhibited both dynamic pausing and fast-running mitochondria motility in both directions (Fig. $7 C$ and $D$ and Movies S13-S15).

Together, these results reveal a pathogenic role for D2 in inducing axonal degeneration in DRG neurons. They further suggest that Bort-induced D2 accumulation caused by Bort exposure may contribute to the DRG damage by a selective inhibition of mitochondria motility.

\section{Discussion}

In this study, we adapted the BIPN model to test the hypothesis that seemingly unrelated CIPN-causing drugs share a pathogenic mechanism by converging on alterations of the tubulin cytoskeleton. In support of this hypothesis, we discovered that acute exposure to the proteasome inhibitor Bort induced accumulation of D2, a marker of hyperstable MTs and the only known irreversible tubulin PTM, in vitro and in vivo. Importantly, an increase in D2 could be measured in a rare human sural nerve biopsy from a cancer patient affected by BIPN, and D2 accumulation was sufficient and necessary for Bort induction of axonopathy and the inhibition of mitochondria motility in DRG neurons.

The innovation and potential of our findings is multifold as we demonstrate that 1) a nontaxane and nonvinca alkaloid compound unexpectedly leads to the neurotoxic accumulation of an irreversible tubulin PTM by affecting MT dynamics, stabilization, and tubulin turnover, providing a strong support for a model of pathogenesis in which CIPN drugs share a pathogenic mechanism converging on tubulin changes; 2) selective D2 accumulation within small unmyelinated fibers, which are preferentially affected in Bort-treated cancer patients (50-52), supports the notion that D2 detection could serve as an early biomarker of BIPN; 3) D2 levels may represent a general hallmark of premature MT and tubulin longevity associated with both acute and slow-developing neurodegeneration, as an increase in glutamylated D2 has recently been reported in the stratum lucidum of a kainate-induced epileptic seizure mouse model and in the hippocampi of postmortem patients with Alzheimer's disease (53); and 4) D2 accumulation affects both axonal viability and mitochondria motility in DRG neurons, underscoring a previously unrecognized role for the tubulin tyrosination/de-tyrosination 
A

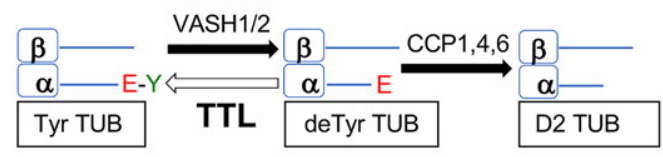

B

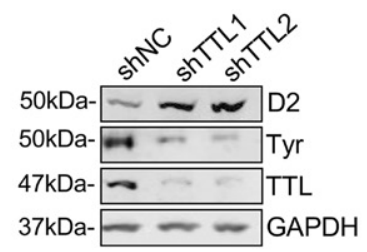

D

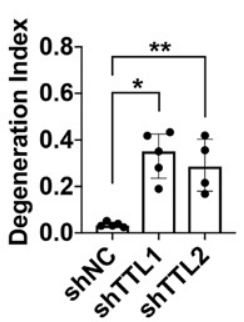

G

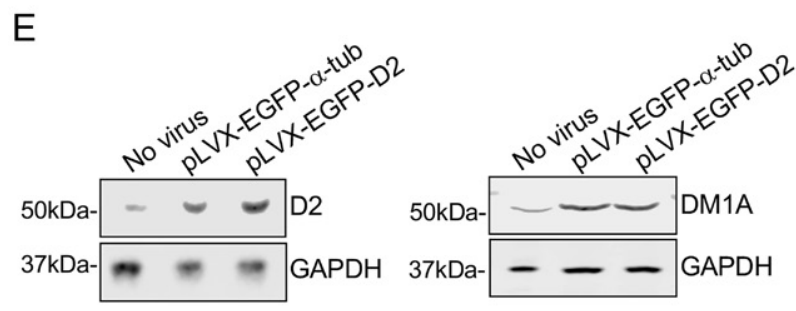

$\mathrm{F}$

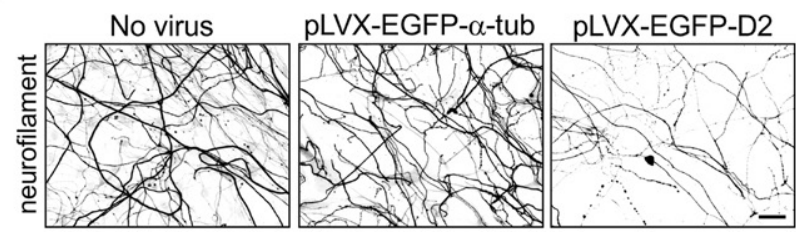

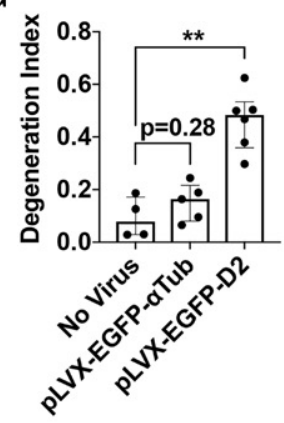

Fig. 5. D2 accumulation is sufficient to drive Bort-induced axonopathy. (A) Schematic of the enzymes involved in D2 generation. (B) Immunoblot analyses of TTL, D2, and tyrosinated tubulin levels in adult DRG neurons (12 DIV) silenced of TTL expression by lentiviral shRNA delivery (5 DIV). (C) Representative IF images of neurofilament staining in DRG neurons (12 DIV) silenced of TTL expression for $7 \mathrm{~d}$ by lentiviral shRNA delivery. (D) Axonal degeneration in DRG neurons treated as in C. Data are pooled from 4 to 5 experiments ( 6 to 16 fields per condition for each experiment). (E) Immunoblot analyses of D2 and $\alpha$-tubulin (DM1A) levels in adult DRG neurons (12 DIV) expressing D2-(pLVX-EGFP- D2) or WT $\alpha$-tubulin (pLVX-EGFP- $\alpha$-tub) (5 DIV). GAPDH, loading control. $(F)$ Representative IF images of neurofilament staining in DRG neurons (12 DIV) transduced with lentiviruses expressing D2-(pLVX-EGFP-D2) or WT $\alpha$-tubulin (pLVX-EGFP- $\alpha$-tub) for $7 \mathrm{~d}$. (G) Axonal degeneration in DRG neurons treated as in $F$. All data in $D$ and $G$ are shown as medians plus interquartile range from 4 to 6 experiments ( 4 to 17 fields per condition for each experiment). Statistical significance was analyzed by Mann-Whitney U test. GAPDH, loading control. shNC, shRNA noncoding control. ${ }^{*} P<0.05,{ }^{*} P<0.01$. Scale bars in $C$ and $F, 50 \mu \mathrm{m}$.

cycle in the control of axonal integrity that may occur through the regulation of mitochondria transport.

We found that in DRG neurons, D2 accumulation correlates with an acute increase in MT stability and a time-dependent buildup of soluble D2. The selective accumulation of soluble D2 is consistent with the proteasome inhibition activity of Bort and the irreversible nature of D2, the half-life of which, in contrast to other tubulin PTMs, is only dependent on degradation.

We explored the mechanism of D2 accumulation on MT polymer and found that Bort acutely affected MT behavior in vitro and in vivo with no substantial alteration of the levels of two rate limiting D2-regulating enzymes, even at time points when accumulation of D2 is already detectable. Unlike cultured DRG neurons and nociceptive neurons of Drosophila larvae in which Bort acutely inhibited dynamic MT behavior, Bort only caused induction of MT growth rates in DRG neurons residing in the skin of adult zebrafish. Either an inhibition of MT dynamics or an increase in MT polymerization alone might lead to MT stabilization, supporting the notion that Bort stabilizes MTs through acute perturbation of MT dynamics in vitro and in vivo. The discrepancy in MT behavior observed in the zebrafish may reflect differences in the intracellular concentration of the drug and/or a consequence of the shielding effect offered by the epithelial layer, which is also affected by bath administration of the drug. We further note that because of imaging limitations imposed by in vitro and in vivo recordings, MT dynamics were measured distally in the axons of the zebrafish and proximally in the axons of cultured DRG neurons and primary dendrites of nociceptive neurons from Drosophila larvae.

In the current study, D2 accumulation was sufficient and necessary to induce axonopathy and affected mitochondria motility in adult DRG neurons. Our results are consistent with published observations in which ablation of TTL correlates with severe defects in neuronal development and failed regeneration of axotomized axons, indicating a harmful role for increased D2 levels in neurite extension $(27,46)$. The findings that Bort led to neurotoxicity and accumulation of both polymeric and soluble D2, while expression of WT $\alpha$-tubulin did not significantly affect axonal integrity or mitochondria motility despite leading to mild D2 accumulation on MTs, suggests that the increase in soluble D2, rather than polymeric, may predominantly contribute to toxicity. 


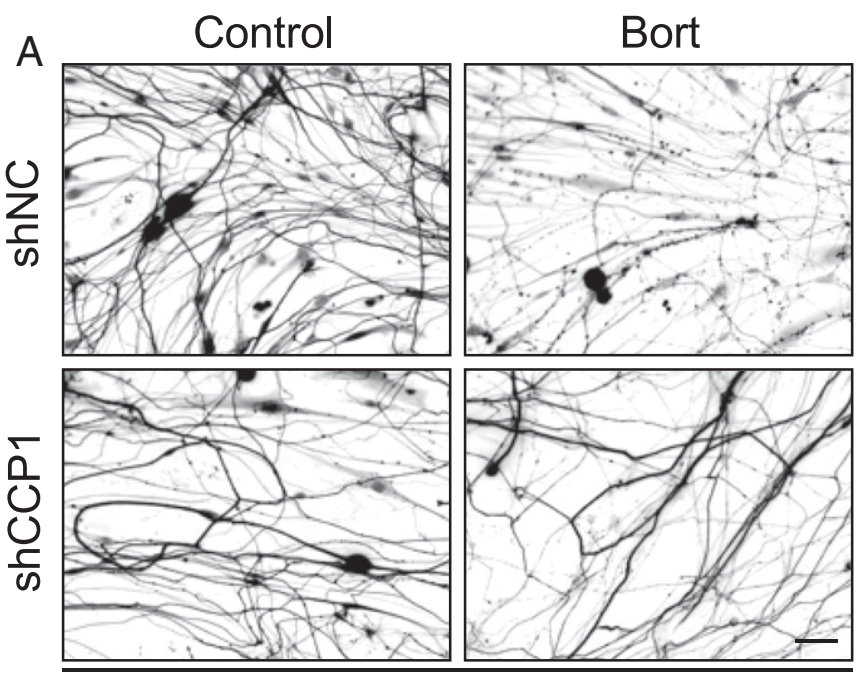

neurofilament

B

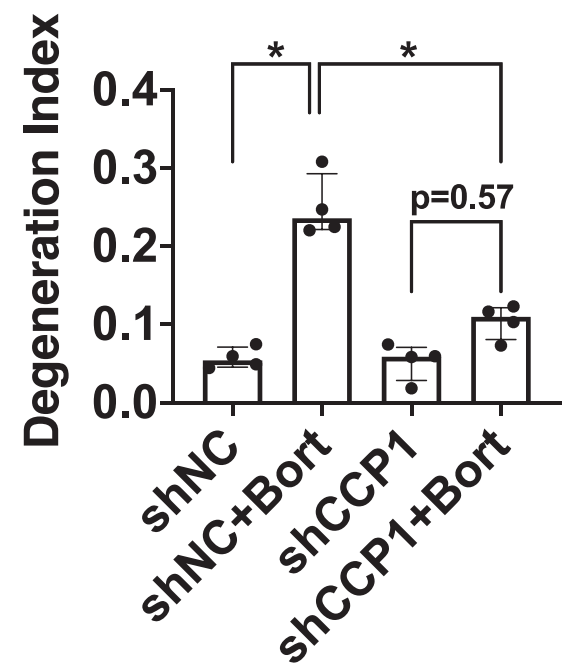

Fig. 6. D2 accumulation is necessary to drive Bort-induced axonopathy. $(A)$ Representative IF images of neurofilament staining in DRG neurons silenced of CCP1 expression at 5 DIV and treated at 12 DIV with $100 \mathrm{nM}$ of Bort for 24 h. Scale bar, $50 \mu \mathrm{m}$. (B) Axonal degeneration in DRG neurons treated as in $A$. Data are from at least 6 to 12 fields per condition ( $n=4$ experiments). Data are shown as medians plus interquartile range, and statistics were analyzed by Mann-Whitney $U$ test. ${ }^{\star} P<0.05$.

Partial CCP1 depletion, to levels that rescue axonal degeneration and cause no detectable changes in polyglutamylated tubulin, antagonized the loss of mitochondria motility induced by Bort. Conversely, D2 overexpression that caused axonal degeneration also promoted inhibition of mitochondria motility. Our findings are consistent with reports of a primary role of loss of mitochondria homeostasis in the disruption of axonal integrity and in the onset of CIPN (54). Interestingly, a link between neurodegeneration and MT hyperglutamylation by complete CCP1 loss of function has been recently reported in cerebellar atrophy observed in CCP1 knockout (KO) mouse models and infants with a defect of the gene encoding CCP1, presumably through regulation of mitochondria motility and fusion $(28,29$, 55). Ablation of CCP1 expression has also been linked to the pausing of migrating cortical interneurons, leading to defects in cortical morphogenesis (56). The contribution of loss of D2 caused by complete CCP1 depletion to the onset of cerebellum neurodegeneration or cortical development, however, has not been addressed. It is possible that compensation from CCP6 and/or regulation of the tyrosination/de-tyrosination cycle may vary among different types of neurons, and that loss of D2 is not as detrimental for neuronal viability as its accumulation. Indeed, we found that acute loss of D2 in cultured adult DRG neurons did not affect neither axonal integrity nor mitochondria motility. Nevertheless, our experiments of CCP1 depletion that did not result in MT hyperglutamylation while significantly inhibiting D2 formation strongly suggest an unexpected role for Bort-induced accumulation of D2 in driving axonal degeneration and defective mitochondria motility in DRG neurons.

At present, we do not have a mechanistic explanation for the selective loss of mitochondria motility caused by D2 accumulation (refer to SI Appendix, Fig. S9 for a schematic of our model). It may be that the irreversible accumulation of a soluble pool of D2 alters the composition of tubulin available for polymerization and in defects in tubulin/MT partitioning. Indeed, subtle changes in tubulin partitioning may impact mitochondria bioenergetics through tubulin interaction with VDACs with indirect consequences on motility $(18,19,57,58)$. On the other hand, D2 may interfere with the adaptor machinery that recruits mitochondria to MT-dependent motors or mitochondria-anchoring proteins, leading to inhibition of motility (59). For example, D2 may increase intracellular $\mathrm{Ca}^{2+}$ levels through an unknown mechanism and induce Miro-dependent dissociation of MT-dependent motors. D2 accumulation may alternatively promote mitochondria localization of endogenous myosin V or syntaphilin, resulting in mitochondria arrest. Further work is necessary to provide insight into this regulation and the cross-talk between D2 and other tubulin PTMs, such as polyglutamylation and acetylation in the control of mitochondria energetics and dynamics. It will be equally crucial to investigate whether D2 accumulation affects responses to pro-survival and pro-death signals that could play a role in CIPN axon degeneration, including regulation of TRPV and $\mathrm{Ca}^{2+}$ channels $(12,13,21,23,60,61)$.

Together, our data provide a strong rationale for pursuing the potential of targeting tubulin modifying enzymes of the tyrosination/de-tyrosination cycle in drug therapies aimed at preventing and/or rescuing axonal injury observed in BIPN.

\section{Materials and Methods}

Reagents. All chemicals were obtained from Sigma-Aldrich unless otherwise noted.

Bortezomib Administration in Rats. Bort (LC Laboratories, Woburn, MA) was formulated in $10 \%$ Tween $80,10 \%$ ethanol, and $80 \%$ sterile saline. The study was divided into two experimental settings. Setting 1 involved acute treatment to test the modifications induced by a single intravenous (i.v.) administration of Bort at $0.20 \mathrm{mg} / \mathrm{kg}$ given as a single dose (and then killed after $24 \mathrm{~h})$. Setting 2 involved chronic treatment to evaluate the neurotoxicity induced by Bort injected at $0.20 \mathrm{mg} / \mathrm{kg}$ i.v. three times per week for $8 \mathrm{wk}$ as previously described (37). For each setting we analyzed untreated animals (the control, or Contr) and Bort-treated groups. The neurophysiological and behavioral tests were performed before the beginning of the treatment period (baseline value) and either $24 \mathrm{~h}$ after a single dose of Bort injection (setting 1) or at the end of $8 \mathrm{wk}$ of Bort treatment (setting 2). All experiments were carried out between 9:00 AM and 4:00 PM.

Primary Antibodies Used for Western Blot or Immunofluorescence Analysis. Detyrosinated tubulin (rabbit, 1:200 immunofluorescence (IF); 1:2,000 Western blot (WB), Millipore), tyrosinated tubulin (rat, 1:400 IF, 1:2,000 WB, Millipore), acetylated tubulin (mouse, 1:300 IF, 1:2,000 WB, Sigma Aldrich), D2 (rabbit, 1:400 IF, 1: 1,000 WB, Millipore), polyglutamylated tubulin (mouse GT335, 1:200 IF or rabbit polyE, 1:2,000 WB, AdipoGen), $\beta$ Ill tubulin (mouse and rabbit, 1:500 IF; 1:2,000 WB, Abcam), $\alpha$-tubulin (mouse DM1A, 1:2,000 WB, Sigma-Aldrich), CCP1 (rabbit, 1:1,000 WB, Proteintech), TTL (rabbit, 1:1,000 WB, Proteintech), GADPH (mouse and rabbit, 1:5,000 WB, Thermo Fisher and Sigma-Aldrich), peripherin (mouse, 1:200 IF, Abcam), and neurofilament (NF-200 chicken on tissue and mouse on DRG neurons, 1:200 IF, 
A

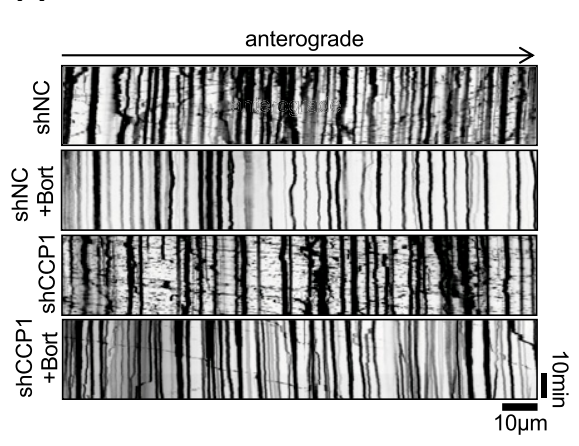

C

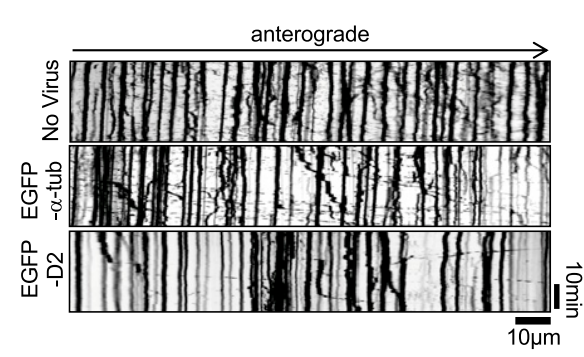

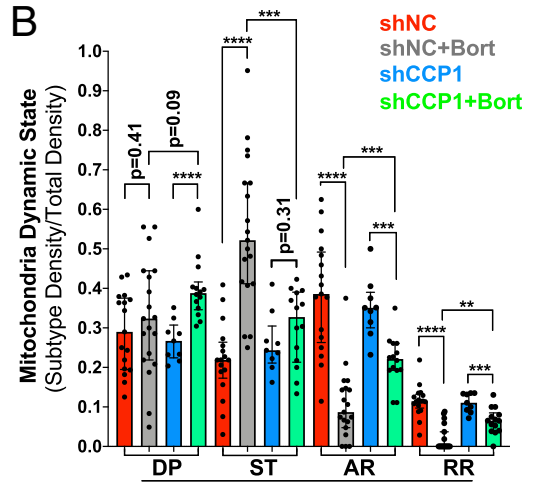

Subtype

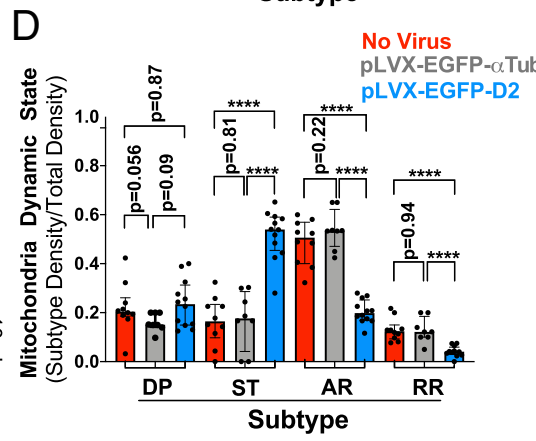

Fig. 7. D2 accumulation is sufficient and necessary to drive defects in mitochondria motility caused by Bort. ( $A$ and $C$ ) Representative kymographs of mitochondria motility in DRG neurons infected with shCCP1 and shNC lentivirus $(A)$ or pLVX-EGFP-D2 and WT- $\alpha$-tubulin lentivirus (C) prior to infection with Mito-DsRed lentivirus and Bort treatment $(A)$. Videos, $10 \mathrm{~s} /$ frame for $30 \mathrm{~min}$. ( $B$ and $D$ ) Quantification of mitochondria dynamic state density in neurons treated as in $A$ and $C$ and expressed as a fraction of total mitochondria density. Dynamic Pause (DP); Stationary (ST); Anterograde Running (AR); Retrograde Running (RR). Data shown in $B$ and $D$ are from 9 to $19(B)$ or 8 to 12 neurites $(B)$ pooled from three independent experiments. All data in $B$ and $D$ are shown as medians plus interquartile range, and statistics were analyzed by Mann-Whitney $U$ test. **P<0.01, ${ }^{* *} P<0.001$, and $* * * * P<0.0001$.

Aves Labs) levels were detected using commercially available specific primary antibodies.

Isolation of Adult DRG Neurons. DRG were dissected from 8- to 10-wk-old C57BL/6J mice in cold Hank's balanced salt solution (HBSS) (Life Technologies) or Dulbecco's Modified Eagle's medium (Life Technologies) and dissociated in $1 \mathrm{mg} / \mathrm{mL}$ Collagenase A for $1 \mathrm{~h}$ at $37{ }^{\circ} \mathrm{C}$, followed by $0.05 \%$ trypsin (Life Technologies) digestion for 3 to $5 \mathrm{~min}$ at $37{ }^{\circ} \mathrm{C}$ and washed with Neurobasal medium (Invitrogen) supplemented with 2\% B-27 (Invitrogen), $0.5 \mathrm{mM}$ glutamine (Invitrogen), fetal bovine serum (FBS), and $100 \mathrm{U} / \mathrm{mL}$ penicillin-streptomycin. DRG neurons were then triturated by repeated gentle pipetting until no clump was visible, and neuronal bodies were resuspended in Neurobasal medium with FBS prior to plating onto 12 well plates (over $18 \mathrm{~mm}$ coverslips) that had been coated overnight with $100 \mu \mathrm{g} /$ $\mathrm{mL}$ poly-D-lysine at $37{ }^{\circ} \mathrm{C}$ and for $1 \mathrm{~h}$ at $37{ }^{\circ} \mathrm{C}$ with $10 \mu \mathrm{g} / \mathrm{mL}$ laminin (Life Technologies). After 30 min, Neurobasal medium, without FBS, was added to the plate. At $4 \mathrm{DIV}$, at least $30 \%$ of media was changed and $10 \mu \mathrm{M}$ AraC was added to media every $4 \mathrm{~d}$

Degeneration Index Measurements in DRG Neurons. Images of an average of 10 random fields of dissociated adult DRG neurons fixed and immunostained with mouse antineurofilament antibodies were acquired using a $20 \times$ objective lens (Olympus IX81) coupled to a monochrome charge-coupled device camera (Sensicam QE; Cooke Corporation). To quantify axonal degeneration, the areas occupied by the axons (total axonal area) and degenerating axons (fragmented axonal area) were measured in the same field from images of DRG neurons. Images were automatically thresholded (global threshold) using a default autothreshold method, binarized, and the frag mented axonal area measured by using the particle analyzer module of ImageJ (size of small fragments $=20$ to 10,000 pixels). Degeneration index was calculated as the ratio between the fragmented axonal area and the total axonal area (62)

Lentiviral Infection of Isolated Adult DRG Neurons. Lentiviral particle delivery technique was employed to infect adult DRG neurons with lentiviral plasmid
DNA or shRNA. Production of lentiviral particles was conducted using the second-generation packaging system as previously described (63). In brief, HEK293T cells were cotransfected with lentiviral plasmid DNA or shRNA and the packaging vectors $\mathrm{PLP} 1, \mathrm{pLP} 2$, and $\mathrm{pLP}-\mathrm{VSV}-\mathrm{G}$ (Thermo Fisher) using the calcium phosphate transfection method. At 24, 36, and $48 \mathrm{~h}$ after transfection, the virus-containing supernatant was collected and the lentiviral particles concentrated ( 800 -fold) by ultracentrifugation $\left(100,000 \times \mathrm{g}\right.$ at $4{ }^{\circ} \mathrm{C}$ for $2 \mathrm{~h})$ prior to aliquoting and storage at $-80^{\circ} \mathrm{C}$.

Plasmid DNA and shRNA. Lentiviral constructs to overexpress WT and D2 tubulins were generated by subcloning of WT and D2 tubulins from WT and D2 pEGFP-tubulin vectors (Clontech) into pLVX lentivector. Briefly, pEGFP-D2 tubulin was generated by a site-directed mutagenesis kit (Agilent) and confirmed by standard Sanger sequencing (Genewiz). Lentiviral WT and D2 tubulins were then generated by digesting pEGFP-WT or D2 tubulin with restriction enzymes, $A f e 1$ and $B a m H 1$, and ligation of the insert into pLVX lentivector. Lentiviral pVL-MitoDsRed and Lamp1-mRFP-FLAG were purchased from Addgene (cat. 44386, 34611). Lentiviral EB3-EGFP was generated by subcloning EB3-EGFP (a gift from Franck Polleux) into pLVX lentivector by Afe1 and Not1 sequential digestions. Two TTL shRNA sequences were purchased from Sigma-Aldrich in pLKO.1 vectors: shTTL1 (TRCN0000191515; sequence: CCG GCA TTC AGA AA GAG TAC TCA ACT CGA GTT GAC TAC TCT TTC TGA ATG CTT TTT TG) and shTTL2 (TRCN0000191227; sequence: CCG GCT CAA AGA ACT ATG GGA AAT ACT CGA GTA TTT CCC ATA GTT CTT TGA GTT TTT TG). The pLKO.1 vector with noncoding (NC) sequence was used as control. The CCP1 shRNA was purchased from SigmaAldrich in PLKO.1 vector (TRCN0000347379; sequence: GTG CCG GAG TGA AAC AGC TTA TTA TCT CGA GAT AAT AAG CTG TTT CAC TCC ATT TTT TG).

MT Fractionation Assays. DRG neurons were treated with vehicle (dimethyl sulfoxide [DMSO]) or $100 \mathrm{nM}$ Bort (LC Laboratories, Woburn, MA) up to $48 \mathrm{~h}$ at $37{ }^{\circ} \mathrm{C}$. At the end of the incubation time, cells were gently washed with warm PHEM $1 \times$ buffer ( $60 \mathrm{mM}$ Pipes, $25 \mathrm{mM}$ Hepes, $10 \mathrm{mM}$ ethylene glycolbis( $\beta$-aminoethyl ether)- $\mathrm{N}, \mathrm{N}, \mathrm{N}^{\prime}, \mathrm{N}^{\prime}$-tetraacetic acid, and $4 \mathrm{mM} \mathrm{MgCl}_{2} ; \mathrm{pH}$ 6.9) once before extraction with $80 \mu \mathrm{L}$ PHEM $1 \times$ buffer, supplemented with 
$0.05 \%$ Triton $\mathrm{X}-100$, protease inhibitor mixture, and $10 \mu \mathrm{M}$ taxol. After $5 \mathrm{~min}$ at $37^{\circ} \mathrm{C}$, the supernatant containing the soluble tubulin fraction was quickly collected in $20 \mu \mathrm{l} 5 \times$ Laemmli buffer and $100 \mu \mathrm{l} 1 \times$ Laemmli buffer was added to each tube to collect the MT pellet (32).

MT Stability Assays. DRG neurons (12 DIV) were treated with vehicle (DMSO) or $100 \mathrm{nM}$ Bort up to $6 \mathrm{~h}$ before addition of $1 \mu \mathrm{g} / \mathrm{mL}$ nocodazole for $45 \mathrm{~min}$. At the end of the incubation time, DRG neurons were gently washed with warm PHEM $1 \times$ buffer once before extraction with PHEM $2 \times$ buffer, supplemented with $0.05 \%$ Triton $\mathrm{X}-100$, protease inhibitor mixture, and $10 \mu \mathrm{M}$ taxol. After $5 \mathrm{~min}$ at $37^{\circ} \mathrm{C}$, a matching volume of $2 \times$ fixative buffer ( $8 \%$ PFA and $0.2 \%$ glutaraldehyde in $1 \times$ PHEM) was added dropwise to the coverslips, and cells were incubated for another $30 \mathrm{~min}$ at $37^{\circ} \mathrm{C}$. DRG neurons were finally washed with PBS $1 \times$ and processed for IF labeling. All images were analyzed using ImageJ software by measuring mean $\beta$ III intensity on major proximal neurites (within $100 \mu \mathrm{m}$ from the cell body) (32).

MT Dynamics Assays in Cultured DRG Neurons. DRG neurons (6 DIV) grown on MatTek dishes were infected with EB3-EGFP lentivirus for $5 \mathrm{~d}$ and live-cell imaging of EB3 comets was performed in complete HBSS media (HBSS, $30 \mathrm{mM}$ glucose, $1 \mathrm{mM} \mathrm{CaCl}_{2}, 1 \mathrm{mM} \mathrm{MgSO}{ }_{4}, 4 \mathrm{mM} \mathrm{NaHCO}_{3}$, and $2.5 \mathrm{mM}$ Hepes; $\mathrm{pH}$ 7.4) using an epifluorescence microscope (Nikon Ti) with a temperature-controlled $\left(37^{\circ} \mathrm{C}\right) \mathrm{CO}_{2}$ incubator ( $2 \mathrm{~s} /$ frame for $5 \mathrm{~min}$ ) at $0,1,2$, 6 , and $24 \mathrm{~h}$ after treatment with $100 \mathrm{nM}$ Bort. To better visualize the comets and reduce background, the mean projection of each time-lapse was subtracted from each frame. Kymographs were generated by drawing a region of $100 \mu \mathrm{m}$ from the cell body using ImageJ or Metamorph software. Parameters describing MT dynamics were defined as follows: rescue/nucleation frequency is the number of rescue or nucleation events per micrometer squared per minute; catastrophe frequency is the number of full tracks/total duration of growth; comet density is the number of comets per micrometer squared per minute; growth length is the comet movement length in micrometers; comet lifetime is the duration of growth in seconds; and growth rate is the growth length/comet lifetime $(32,64)$.

MT Dynamics Assays in Zebrafish Nerve Endings. Adult zebrafish (Danio rerio) were raised in an Aquaneering fish habitat set to $\mathrm{pH} 7.5,400-$ to $500-\mu \mathrm{S}$ salinity, and $28{ }^{\circ} \mathrm{C}$ with $10 \%$ water exchanges daily. Fish used in the experiment were in a Nacre mutant background and doubly heterozygous for the transgenic insertions P2 $\times$ 3.LexA.LexAop.EB3-GFP and P2 $\times$ 3.LexA.LexAop.mCherry that we used as a cytosolic filler to visualize nerve endings. The mCherry-labeled strain was a generous gift from Alvaro Sagasti of the University of California, Los Angeles, and the EB3-GFP fish were generated in the Rolls laboratory using the gateway system and tol2 kit as previously published by Feng et al. (65). After the first imaging session, fish were housed in standard mating cages, one fish per cage with no divider, and their water exchanged every $24 \mathrm{~h}$ with fresh system water with $0.01 \%$ DMSO and $1.3 \mu \mathrm{M}$ Bort or $0.01 \%$ DMSO for vehicle-treated control fish. Fish were mounted in $2 \%$ low-melt agarose and anesthetized with $0.012 \%$ tricaine but otherwise were intubated and recovered as described by Rasmussen et al. (66). Imaging was performed on a Zeiss LSM800 inverted microscope using a 20 $0.8 \mathrm{NA}$ air objective. Time series of images were taken with a zoom of $5.3,512 \times 512$ pixel size and a $4-\mu \mathrm{s}$ pixel dwell time for a frame time of $1.3 \mathrm{~s}$. Kymographs were generated using the multikymograph feature of ImageJ. Parameters describing MT dynamics were applied as in cultured DRG neurons. All zebrafish experiments were performed after receiving approval from the Penn State Institutional Animal Care and Use Committee, and guidelines on euthanasia and pain management were followed.

MT Dynamics in Drosophila Larvae. Preparation of larvae for live imaging was performed as previously described (67). Briefly, a drop of fresh $3 \%$ agarose in $\mathrm{ddH}_{2} \mathrm{O}$ was placed on a slide and dried overnight at $65^{\circ} \mathrm{C}$ before use. Single larvae were cleaned, placed on the dried agarose patch, and gently squeezed by a coverslip (size \#1, Thorlab). This sandwich was held in place with sticky tape. Once mounted, the larva was imaged within $45 \mathrm{~min}$. Live imaging was performed using a Yokogawa W1 spinning disk confocal microscope mounted on a Nikon Ti2-E stand (40x objective, water, $1.15 \mathrm{NA}$,

1. S. B. Park et al., Chemotherapy-induced peripheral neurotoxicity: A critical analysis. CA Cancer J. Clin. 63, 419-437 (2013).

2. G. Cavaletti, P. Marmiroli, Chemotherapy-induced peripheral neurotoxicity. Nat. Rev. Neurol. 6, 657-666 (2010).

3. A. A. Argyriou, J. Bruna, P. Marmiroli, G. Cavaletti, Chemotherapy-induced peripheral neurotoxicity (CIPN): An update. Crit. Rev. Oncol. Hematol. 82, 51-77 (2012) and CFI60 Plan Fluor). Nociceptive neuron-specific EB1-GFP expression was used to identify ROls. Two to four neurons were selected per animal, and the proximal area of neurons were imaged at 2 s/frame. Focus was manually adjusted when necessary to counter minor movements of the animal. Images were collected for up to $6 \mathrm{~min}$, depending on the stability of the mounted larva. All images were randomized and blinded before any correction or quantification. Images were corrected for minor movements using the Stackreg plugin for Fiji software. Kymographs were generated by using Fiji's plugins tracing the primary neurites of larvae sensory neurons using a seg mented line tool. Parameters describing MT dynamics were applied as in cultured DRG neurons.

Mitochondria and Lysosome Motility Assays. Dissociated adult DRG neurons were first transduced at $12 \mathrm{~h}$ after plating with shCCP1, shNC, or pLVX-D2 and pLVX-WT- $\alpha$-tubulin lentiviruses and $24 \mathrm{~h}$ later with Mito-DsRed lentivirus. To track lysosome motility, neurons were transduced at $24 \mathrm{~h}$ after plating with Lamp1-mRFP-FLAG lentivirus. When treated at 4 DIV neurons were incubated with vehicle (DMSO) or $100 \mathrm{nM}$ Bort and live imaged at 5 DIV using an epifluorescence microscope (Nikon Ti), equipped with a temperaturecontrolled $\left(37{ }^{\circ} \mathrm{C}\right) \mathrm{CO}_{2}$ incubator. Proximal regions from intact axons (within $100 \mu \mathrm{m}$ from the cell body) were selected for imaging, and movies were acquired at $10 \mathrm{~s} /$ frame for $30 \mathrm{~min}$ to track mitochondria and lysosomes. Kymographs were generated manually with a Fiji's plugin by tracing the proximal axon using a segmented line tool. Mitochondria and lysosome dynamic states were identified on kymographs and classified as follows: lysosomes and mitochondria were defined as stationary (ST) if fixed in an immobile state for the entire length of the movie. Dynamic mitochondria or lysosomes were subclassified as dynamic pausing, if showing persistent and short oscillatory movements in either direction (approximately $<5 \mu \mathrm{m}$ ), or AR and RR running if moving for short or longer distances in either direction (approximately $>5 \mu \mathrm{m}$ ). The number of different movement states per movie was scored and quantified on kymographs. The density of each dynamic state was reported as a percentage of total density to normalize for fluctuations in total numbers.

Statistics. Data distributions that did not pass the Shapiro-Wilk normality test are shown as medians plus interquartile range, and statistical significance was analyzed by nonparametric unpaired two-tailed Mann-Whitney $U$ test or Kruskal-Wallis with Dunn's multiple comparisons test. Data distributions that passed the normality test are shown as means \pm SEM, and statistical significance was analyzed by parametric one-way ANOVA for multiple comparisons (GraphPad Prism 8.0.0 software). For analyses of MT growth rates, length of growth, and comet lifetimes, when comparing groups, we assumed that each EB comet measure is an independent observation. All experiments were repeated at least three times unless stated otherwise. Detailed description of $n$ values are included in each figure legend, and individual $P$ values for each comparison are listed in SI Appendix, Table S1.

Data Availability. All study data are included in the article and supporting information.

ACKNOWLEDGMENTS. This project was funded by a Thompson Family Foundation (TFFI) award to F.B. and G.C., a TFFI award to W.B.G., RO1AG050658 (NIH/National Institute on Aging) and R21NS120076 (NIH/ NINDS) awards to F.B., and a PRIN-2017FJC3-004 (MIUR) to G.C. and M.E.P We are grateful to Gregg G. Gundersen for stimulating discussions and access to his microscopes. We thank Giuseppe Tosto for advising on statistical analysis of the data and Samie Jules for technical assistance in the analysis of the experiments with adult DRG neurons. Our gratitude goes to Annalisa Canta, Alessia Chiorazzi, Elisa Ballarini, and Virginia Rodriguez for technical assistance in rat behavior and neurophysiology experiments and to Luke Hammond for optimizing conditions for EB1 imaging in Drosophila. We are grateful to Terry Hafer for providing the sample preparation protocol for live imaging of Drosophila larvae, Robert Cudmore (Johns Hopkins) for Python codes for blinding files collected in Drosophila experiments, and the Zuckerman Institute's Cellular Imaging platform for instrument use and technical advice for Drosophila imaging. We thank Elena Bianchetti and Markus D. Siegelin for assisting us with caspase cleavage assays.

4. W. Grisold, G. Cavaletti, A. J. Windebank, Peripheral neuropathies from chemotherapeutics and targeted agents: Diagnosis, treatment, and prevention. Neuro-oncol. 14 (suppl. 4), iv45-iv54 (2012)

5. G. Cavaletti, P. Alberti, B. Frigeni, M. Piatti, E. Susani, Chemotherapy-induced neuropathy. Curr. Treat. Options Neurol. 13, 180-190 (2011).

6. G. Cavaletti, G. Nicolini, P. Marmiroli, Neurotoxic effects of antineoplastic drugs: The lesson of pre-clinical studies. Front. Biosci. 13, 3506-3524 (2008). 
7. J. C. Fehrenbacher, Chemotherapy-induced peripheral neuropathy. Prog. Mol. Biol. Transl. Sci. 131, 471-508 (2015).

8. J. Ferrier, V. Pereira, J. Busserolles, N. Authier, D. Balayssac, Emerging trends in understanding chemotherapy-induced peripheral neuropathy. Curr. Pain Headache Rep. 17, 364 (2013).

9. A. Canta, A. Chiorazzi, G. Cavaletti, Tubulin: A target for antineoplastic drugs into the cancer cells but also in the peripheral nervous system. Curr. Med. Chem. 16, 1315-1324 (2009)

10. E. L. Gornstein, T. L. Schwarz, Neurotoxic mechanisms of paclitaxel are local to the distal axon and independent of transport defects. Exp. Neurol. 288, 153-166 (2017).

11. L. J. Leandro-García et al., Regulatory polymorphisms in $\beta$-tubulin Ila are associated with paclitaxel-induced peripheral neuropathy. Clin. Cancer Res. 18, 4441-4448 (2012).

12. W. T. Harkcom et al., NAD+ and SIRT3 control microtubule dynamics and reduce susceptibility to antimicrotubule agents. Proc. Natl. Acad. Sci. U.S.A. 111, E2443-E2452 (2014).

13. S. Geisler et al., Prevention of vincristine-induced peripheral neuropathy by genetic deletion of SARM1 in mice. Brain 139, 3092-3108 (2016)

14. J. T. Smyth, W. I. DeHaven, G. S. Bird, J. W. Putney Jr, Role of the microtubule cytoskeleton in the function of the store-operated Ca2+ channel activator STIM1. J. Cell Sci. 120, 3762-3771 (2007).

15. C. Janke, M. Kneussel, Tubulin post-translational modifications: Encoding functions on the neuronal microtubule cytoskeleton. Trends Neurosci. 33, 362-372 (2010)

16. B. Lacroix et al., Tubulin polyglutamylation stimulates spastin-mediated microtubule severing. J. Cell Biol. 189, 945-954 (2010).

17. L. Cassimeris et al., Fueled by microtubules: Does tubulin dimer/polymer partitioning regulate intracellular metabolism? Cytoskeleton (Hoboken) 69, 133-143 (2012).

18. T. K. Rostovtseva, S. M. Bezrukov, VDAC inhibition by tubulin and its physiological implications. Biochim. Biophys. Acta 1818, 1526-1535 (2012).

19. K. L. Sheldon, P. A. Gurnev, S. M. Bezrukov, D. L. Sackett, Tubulin tail sequences and post-translational modifications regulate closure of mitochondrial voltage dependent anion channel (VDAC). J. Biol. Chem. 290, 26784-26789 (2015)

20. S. Chakraborti, K. Natarajan, J. Curiel, C. Janke, J. Liu, The emerging role of the tubulin code: From the tubulin molecule to neuronal function and disease. Cytoskeleton (Hoboken) 73, 521-550 (2016)

21. Y. Fukuda, Y. Li, R. A. Segal, A mechanistic understanding of axon degeneration in chemotherapy-induced peripheral neuropathy. Front. Neurosci. 11, 481 (2017).

22. G. Landouré et al., Mutations in TRPV4 cause charcot-marie-tooth disease type $2 \mathrm{C}$. Nat. Genet. 42, 170-174 (2010).

23. C. Goswami, TRPV1-tubulin complex: Involvement of membrane tubulin in the reg ulation of chemotherapy-induced peripheral neuropathy. J. Neurochem. 123, 1-13 (2012)

24. C. O. Wong et al., A TRPV channel in Drosophila motor neurons regulates presynaptic resting Ca2+ levels, synapse growth, and synaptic transmission. Neuron 84, 764-777 (2014).

25. K. Rogowski et al., A family of protein-deglutamylating enzymes associated with neurodegeneration. Cell 143, 564-578 (2010).

26. Y. Cho, V. Cavalli, HDAC signaling in neuronal development and axon regeneration Curr. Opin. Neurobiol. 27, 118-126 (2014).

27. W. Song, Y. Cho, D. Watt, V. Cavalli, Tubulin-tyrosine ligase (TTL)-mediated increase in tyrosinated $\alpha$-Tubulin in injured axons is required for retrograde injury signaling and axon regeneration. J. Biol. Chem. 290, 14765-14775 (2015).

28. V. Shashi et al.; Undiagnosed Diseases Network, Loss of tubulin deglutamylase CCP1 causes infantile-onset neurodegeneration. EMBO J. 37, e100540 (2018)

29. M. M. Magiera et al., Excessive tubulin polyglutamylation causes neurodegeneration and perturbs neuronal transport. EMBO J. 37, e100440 (2018)

30. S. Lin, N. A. Sterling, I. P. Junker, C. T. Helm, G. M. Smith, Effects of $\alpha$ TAT1 and HDAC5 on axonal regeneration in adult neurons. PLoS One 12, e0177496 (2017).

31. L. Van Helleputte et al., Inhibition of histone deacetylase 6 (HDAC6) protects against vincristine-induced peripheral neuropathies and inhibits tumor growth. Neurobiol. Dis. 111, 59-69 (2018)

32. X. Qu et al., Stabilization of dynamic microtubules by mDia1 drives Tau-dependent A $\beta_{1-42}$ synaptotoxicity. J. Cell Biol. 216, 3161-3178 (2017).

33. S. J. Morley et al., Acetylated tubulin is essential for touch sensation in mice. elife $\mathbf{5}$, e20813 (2016).

34. S. Geisler et al., Gene therapy targeting SARM1 blocks pathological axon degeneration in mice. J. Exp. Med. 216, 294-303 (2019).

35. G. E. B. Wright et al.; Canadian Pharmacogenomics Network for Drug Safety Consortium, Pharmacogenomics of vincristine-induced peripheral neuropathy implicates pharmacokinetic and inherited neuropathy genes. Clin. Pharmacol. Ther. 105, 402-410 (2019)

36. C. Picci et al., HDAC6 inhibition promotes $\alpha$-tubulin acetylation and ameliorates CMT2A peripheral neuropathy in mice. Exp. Neurol. 328, 113281 (2020).
37. C. Meregalli et al., Evaluation of tubulin polymerization and chronic inhibition of proteasome as citotoxicity mechanisms in bortezomib-induced peripheral neuropathy. Cell Cycle 13, 612-621 (2014).

38. N. P. Staff et al., Bortezomib alters microtubule polymerization and axonal transport in rat dorsal root ganglion neurons. Neurotoxicology 39, 124-131 (2013).

39. L. Paturle-Lafanechère et al., Characterization of a major brain tubulin variant which cannot be tyrosinated. Biochemistry 30, 10523-10528 (1991).

40. L. Paturle-Lafanechère et al., Accumulation of delta 2-tubulin, a major tubulin variant that cannot be tyrosinated, in neuronal tissues and in stable microtubule assemblies. J. Cell Sci. 107, 1529-1543 (1994).

41. I. Berezniuk et al., Cytosolic carboxypeptidase 1 is involved in processing $\alpha$ - and $\beta$-tubulin. J. Biol. Chem. 287, 6503-6517 (2012).

42. J. Nieuwenhuis et al., Vasohibins encode tubulin detyrosinating activity. Science $\mathbf{3 5 8}$ 1453-1456 (2017).

43. C. Aillaud et al., Vasohibins/SVBP are tubulin carboxypeptidases (TCPs) that regulate neuron differentiation. Science 358, 1448-1453 (2017).

44. J. Nieuwenhuis, T. R. Brummelkamp, The tubulin detyrosination cycle: Function and enzymes. Trends Cell Biol. 29, 80-92 (2019).

45. K. Ersfeld et al., Characterization of the tubulin-tyrosine ligase. J. Cell Biol. 120 , 725-732 (1993).

46. C. Erck et al., A vital role of tubulin-tyrosine-ligase for neuronal organization. Proc. Natl. Acad. Sci. U.S.A. 102, 7853-7858 (2005).

47. A. E. Prota et al., Structural basis of tubulin tyrosination by tubulin tyrosine ligase. J. Cell Biol. 200, 259-270 (2013).

48. M. E. Goldstein, S. B. House, H. Gainer, NF-L and peripherin immunoreactivities define distinct classes of rat sensory ganglion cells. J. Neurosci. Res. 30, 92-104 (1991).

49. L. Guo et al., Editor's highlight: Multiparametric image analysis of rat dorsal root ganglion cultures to evaluate peripheral neuropathy-inducing chemotherapeutics. Toxicol. Sci. 156, 275-288 (2017).

50. V. A. Carozzi et al., Bortezomib-induced painful peripheral neuropathy: An electrophysiological, behavioral, morphological and mechanistic study in the mouse. PLOS One 8, e72995 (2013)

51. M. Bechakra et al., Clinical, electrophysiological, and cutaneous innervation changes in patients with bortezomib-induced peripheral neuropathy reveal insight into mechanisms of neuropathic pain. Mol. Pain 14, 1744806918797042 (2018).

52. R. Velasco, P. Alberti, J. Bruna, D. Psimaras, A. A. Argyriou, Bortezomib and other proteosome inhibitors-induced peripheral neurotoxicity: From pathogenesis to treatment. J. Peripher. Nerv. Syst. 24 (suppl. 2), S52-S62 (2019).

53. $\mathrm{H}, \mathrm{T}, \mathrm{Vu}, \mathrm{H}$. Akatsu, $\mathrm{Y}$. Hashizume, $\mathrm{M}$. Setou, $\mathrm{K}$. Ikegami, Increase in $\alpha$-tubulin modifications in the neuronal processes of hippocampal neurons in both kainic acidinduced epileptic seizure and Alzheimer's disease. Sci. Rep. 7, 40205 (2017).

54. A. Canta, E. Pozzi, V. A. Carozzi, Mitochondrial dysfunction in chemotherapy-induced peripheral neuropathy (CIPN). Toxics 3, 198-223 (2015).

55. S. Gilmore-Hall et al., CCP1 promotes mitochondrial fusion and motility to prevent Purkinje cell neuron loss in pcd mice. J. Cell Biol. 218, 206-219 (2019).

56. C. G. Silva et al., Cell-intrinsic control of interneuron migration drives cortical morphogenesis. Cell 172, 1063-1078.e19 (2018)

57. E. N. Maldonado et al., Voltage-dependent anion channels modulate mitochondrial metabolism in cancer cells: Regulation by free tubulin and erastin. J. Biol. Chem. 288 , 11920-11929 (2013).

58. A. Rovini, Tubulin-VDAC interaction: Molecular basis for mitochondrial dysfunction in chemotherapy-induced peripheral neuropathy. Front. Physiol. 10, 671 (2019).

59. T. L. Schwarz, Mitochondrial trafficking in neurons. Cold Spring Harb. Perspect. Biol. 5, a011304 (2013).

60. M. Quartu et al., Bortezomib treatment produces nocifensive behavior and changes in the expression of TRPV1, CGRP, and substance $P$ in the rat DRG, spinal cord, and sciatic nerve. BioMed Res. Int. 2014, 180428 (2014).

61. S. E. Pease-Raissi et al., Paclitaxel reduces axonal Bclw to initiate $I_{3} R 1$-dependent axon degeneration. Neuron 96, 373-386.e6 (2017)

62. J. Gerdts, Y. Sasaki, B. Vohra, J. Marasa, J. Milbrandt, Image-based screening identifies novel roles for IkappaB kinase and glycogen synthase kinase 3 in axonal degeneration. J. Biol. Chem. 286, 28011-28018 (2011).

63. J. Liu, S. Pasini, M. L. Shelanski, L. A. Greene, Activating transcription factor 4 (ATF4) modulates post-synaptic development and dendritic spine morphology. Front. Cell. Neurosci. 8, 177 (2014)

64. T. Stepanova et al., History-dependent catastrophes regulate axonal microtubule behavior. Curr. Biol. 20, 1023-1028 (2010)

65. C. Feng et al., Patronin-mediated minus end growth is required for dendritic microtubule polarity. J. Cell Biol. 218, 2309-2328 (2019).

66. J. P. Rasmussen, N. T. Vo, A. Sagasti, Fish scales dictate the pattern of adult skin innervation and vascularization. Dev. Cell 46, 344-359.e4 (2018).

67. L. Chen et al., Mitochondria and caspases tune nmnat-mediated stabilization to promote axon regeneration. PLoS Genet. 12, e1006503 (2016). 